\title{
Proanthocyanidin Metabolism, a mini review
}

\author{
Y.Y. Choy and A.L. Waterhouse* \\ Viticulture and Enology, University of California, Davis, CA, USA
}

\begin{abstract}
There is emerging evidence suggesting that consumption of beverage and food rich in polyphenol may offer protective effects against various neurodegenerative, cardiovascular diseases and cancers. Proanthocyanidins (PACs) are one of the most abundant polyphenol in human diets, but also one of the least absorbed polyphenol mostly due to their size and structure complexity. PACs or condensed tannins are oligomers and polymers of monomeric unit flavan-3-ol (+)-catechin or (-)-epicatechin. To date, the absorption and metabolism of PACs are still remains largely unknown. The aim of this mini review was to highlight the absorption and metabolism of PACs, their effect in the gut and sample preparation for analysis. Ultimately, the potential bioactivities derived from the interaction between PACs metabolites and the gut microbiota warrants further investigation.
\end{abstract}

Keywords: Proanthocyanidins, phenolic acids, metabolism, colon

\section{Introduction}

Polyphenols are among the ubiquitous constituents of foods of plant origins and are widely distributed throughout the plant kingdom. Polyphenols can be categorized into different groups such as flavonoids, phenolic acids, stilbenes and lignans. The flavonoids consists of 2 aromatic rings (A and B) that are link with a heterocyclic five-membered ring that includes an oxygen atom (ring $\mathrm{C}$ ), and divided into 6 subclasses: flavonols, flavones, isoflavones, flavanones, anthocyanidins, and flavanols (catechins and proanthocyanidins) [22]. Proanthocyanidins (PACs), a subclass of flavonoids, are among the most abundant polyphenol present in human diets. The dietary intake of PACs in the U.S. was estimated to be about $100 \mathrm{mg} / \mathrm{d}$, falling into the following size categories (percentage of total), monomers (22\%), dimers (16\%), trimers (5\%), 4-6 mers (15\%), 7-10 mers (11\%), and polymers $(30 \%)$, and PACs can be found in various foods and beverages including grape seeds, pine bark, apple, peach, pear, berries, cocoa, tea, wine and beer [49]. PACs are considered one of the most complex subclass of flavonoids, consisting of monomers, oligomers or polymers of (-)-epicatechin or $(+)$-catechin and

\footnotetext{
*Corresponding author: A.L. Waterhouse, Viticulture and Enology, University of California, Davis, CA 95616, USA. E-mail: alwaterhouse@ucdavis.edu.
}

derived subunits. PACs that consists exclusively of (-)-epicatechin, (+)-catechin units, are known as procyanidins because only cyanidin is released in acid, and comprised the largest class of PACs, while those with gallocatechin units release delphinidin. The size or molecular weight of PACs can be expressed as mean degree of polymerization ( $\mathrm{mDP}$ ). The major constituents of grapes are monomeric (-)-epicatechin, (+)-catechin, epicatechin-3-O-gallate (more abundant in seeds than in skins) and (-)-epigallocatechin (only found in skins) [11]. Therefore, grape seed tannins are partially galloylated procyanidins. The dimeric procyanidins are often referred as B-type (B1, B2, B3 B4 and B5) or A-type (A1 and A2) and the trimeric procyandins as $\mathrm{C}$-type ( $\mathrm{C} 1$ and $\mathrm{C} 2$ ). The most ubiquitous PACs in foods are the B-type, formed by oxidative coupling between the (C4-C8) bond and to a lesser extent through a (C4-C6) bond. The less common is the A-type procyanidins which have an additional ether bond through a (C2-O-C7) bond or a less abundant (C2-O-C5) bond [48].

\section{Absorption and metabolism data of oligomeric and polymeric PACs}

PACs are known to be poorly absorbed in the gut due to their polymeric nature and high molecular 
weight. Thus, polyphenols are the only dietary antioxidants that are present in the colon at levels up to a few hundred micromoles per liter, given that vitamins $\mathrm{C}$ and $\mathrm{E}$ are absorbed through the upper intestines [22]. This limitation on their absorption may result in a direct beneficial activity in the gastrointestinal (GI) tract. Physiologically phenolics including PACs are treated as xenobiotics, and the small intestine is the primary site for glucuronidation, occurring in luminal part of the endoplasmic reticulum through uridine 5'-diphosphate glucuronosyltransferases (UGTs) while sulfation and methylation occur in the liver via cytosol sulfotransferases (SULT) and catecholO-methyltransferase (COMT) [27]. Before entering the blood stream, the monomeric or dimeric flavan3-ols are either absorbed in the small intestine or transported back to the liver and undergo phase II metabolism, producing glucuronide, sulfate and/or methylated metabolites. Through enterohepatic recirculation, these conjugated compounds may recycle back to the small intestines via bile excretion. It has been estimated that more than $90 \%$ of ingested polyphenols are not absorbed in the small intestine and thus, remain in the colon at high concentration [7]. In the colon, the unabsorbed compounds are extensively metabolized by gut microbiota to produce smaller molecules, including simple phenolic acids such as hydroxybenzoic acid, hydroxyphenylacetic acid, hydroxyphenylpropinoic acid, hyrdroxyphenylvaleric acid or hydroxycinnamic acids, with hydroxylation mostly occurring at meta position [1, 23]. These metabolites can be absorbed or may be conjugated in the liver before being eliminated in the urine. In some cases, the amount of the simple metabolites is significant, suggesting that these metabolites may have important functions [39].

Monomeric flavan-3-ols, including (+)-catechin and (-)-epicatechin, has been previously reported to be absorbed in humans and animals, either as parent compounds or conjugated metabolites following the consumption of dietary PACs [3, 50]. While studies of the metabolism and absorption of monomeric flavan-3-ols are numerous, investigations on the fate of oligomeric and polymeric PACs are controversial, being mostly focused on the fate of dimeric PACs. The quantification and identification of PACs dimers and trimers in plasma or urine following the ingestion of apples [37], cocoa [16, 46] and GSE [30, 35, 36] PACs has been demonstrated. The presence of these oligomeric PACs in plasma suggested that they were absorbed and metabolized similarly to monomeric flavan-3-ol. Low level of PACs dimer B2 has been reported in human urine and plasma after consumption of cocoa PACs $[16,46]$ while dimer B1 was detected in human plasma following ingestion of GSE PACs [35]. The absorption of dimers were estimated to be 100 fold lower than the monomer [16]. PAC dimers and trimers have been detected in the rats urine fed GSE [44]. Recently, Serra et. al have quantified and identified intact PACs dimers and trimers in rats plasma with the concentration ranging from $0.85-8.55 \mu \mathrm{M}$ [36]. The absorption of A-type procyanidins were less studied compared to the B-type. Nevertheless, it has been reported that A-type procyanidins (A1 and A2) were absorbed from small intestines in rats and they were better absorbed than dimer B2 [1, 2]. Future study should be focused on these A-type procyanidins since it has been shown to have protective effects against cardiovascular diseases [21] and an effective uropathogenic bacterial anti adhesion activity [17]. It has been reported that apple PAC oligomers up to pentamers were detected in rat plasma $2 \mathrm{~h}$ after oral administration, [37] however, the administration dose was rather high, $1 \mathrm{~g} / \mathrm{kg}$ body and may not be physiologically relevant. Studies from Rios et.al showed that cocoa PACs were stable in the stomach and reached in the small intestine, and available for absorption or metabolism [32]. A recent feeding study of apple juice to ileostomists have shown that most polyphenols remained unchanged at the large intestine but extensively metabolized by gut microbiota in the colon to various phenolic acids [19]. Due to low absorption of intact PACs in the human colon, microbial metabolism is likely has a major role in colonic health and the potential biological effects are attributed to these gut microbiota metabolites. (reviewed in [8]) In in vitro studies, it has been reported that human gut microbiota degrades PACs to low molecular weight phenolic compounds, [9] suggesting that absorption of the PACs parent compounds is unlikely. Urpi-Sarda et al. have detected various microbial phenolic metabolites and phenylvalerolactones in human and rats urine after cocoa PACs consumption [46].

\section{Colonic microbial metabolism of PACs}

There is no consensus on the absorption and metabolism of PACs thus far, although colon is 


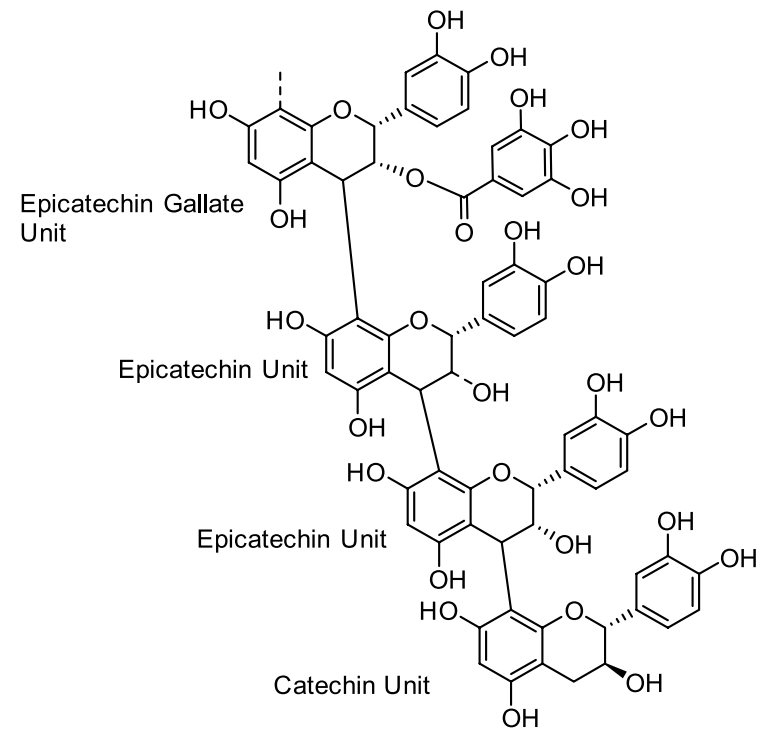

Fig. 1. Sample grape proanthocyanidin tetramer shown with bond extension to additional subunits.

being recognized as an important biotransformation site of these PACs by the gut microbiota. Therefore, the PACs are degraded by either intestinal enzymes or the gut microbiota before they can be absorbed. The potential bioactivities of PACs are attributed to the colonic degradation products, including phenolic acids and valerolactones. Numerous in vivo studies have showed the presence of microbial derived phenolic metabolites in urine after intake of PACs-rich diets. Some of the main urinary microbial phenolic acids found were mono- and di-hydroxy phenylpropionic acids and phenylacetic acids, along with hydroxyhippuric acids [27]. Gonthier et al. found an increase in 3-hydroxyphenylacetic and 3,4dihydroxyphenylacetic acids in urine after the intake of procyanidin B3 in rats [13]. Similarly, Urpi-Sarda et al. reported a significant increase in the urinary level of 3-hydroxyphenylacetic, 3,4-dihydroxyphenylacetic acids and 5-(3', $4^{\prime}$-dihydroxyphenyl)- $\gamma$-valerolactone after regular consumption of cocoa [46]. The main microbial metabolites detected in rat urine after fed with red wine polyphenols were 3hydroxyphenylpropionic acid, 3-hydroxybenzoic acid and 3-hydroxyhippuric acid [12]. The identification of bacteria to catabolize PACs should be further evaluated for a better understanding of the role of PACs-rich diets and relationship of gut microbiota in promoting overall gut health.

\section{Effect of PACs in the gut}

In general, PACs are thought to be poorly absorbed and their health effects may not require direct absorption in the gut. The large PACs (oligomeric or polymeric) can regulate cell signaling pathways by interacting with cell membrane proteins [5], and protecting intestinal barrier integrity through different mechanisms of action including their antioxidant capacity and anti-inflammation activity.

A study has reported that intact parent PACs (dimerhexamer) were detected in the colonic rat feces after ingesting GSE PACs and these dietary components may contribute to the colon health [6]. This is also in line with a recent finding that detected microbial phenolic metabolites in feces, cecal content and colonic tissue following intake of PACs-rich diets [43].

There is mounting evidence from in vitro and in vivo studies of potential health benefits of PACs consumption linked to protection against colorectal cancer (CRC) [20, 38, 47]. Currently, CRC is the third most diagnosed cancer in the US. Epidemiological and clinical studies have reported that high intake of fats and red meat increases the risk of colorectal cancer (CRC) [41]. The consumption of fruits and vegetables, however, is linked to the decrease risk of CRC and may be due to their high content of flavonoids [41, 42]. Among the flavonoids, PACs ( $\geq 2$ subunits) consumption was found to be inversely related to the decrease risk of CRC in an Italian population [33]. The health effects may be attributable not only from the intact PACs but also their microbial phenolic metabolites that are readily absorbed. Thus, a better understanding of the metabolic fate of oligomeric or polymeric PACs in the colon is essential to understand their potential colonic health benefits.

\section{Challenges of analysis of PACs}

PACs are susceptible to oxidation and highly reactive natural products. Their diverse structure and high molecular size make the identification and quantitation of these compounds challenging. Chromatographic methods with combinations of various mass-spectrometry techniques have been the forefront application used in identification and quantification of metabolites in biological samples.

Sample preparation is a key step during method development, particularly working with complex 
biological matrices since the clean-up procedures can greatly affect the results. A thorough sample preparation is needed to minimize matrix effects and increase instrument sensitivity when quantifying compounds of interest by mass-spectrometry. Different biological matrices may require different clean up procedures to remove interference compounds including protein, carbohydrate, salt, organic and inorganic compounds.

Protein precipitation is one the simplest way to remove proteins in biological samples and it has been used in the first step of sample preparation [29]. Protein precipitation was carried out by adding organic solvents such as acetonitrile to the sample, a solvent that dissolves PACs quite well.

One of the most common cleanup methods used by many investigators was solid phase extraction using $\mathrm{C}_{8}$ or $\mathrm{C}_{18}$ SPE cartridges [29, 36]. During feces sample preparation, $50 \%$ aqueous methanol has been shown to be the best extraction solvent and recovery as elution solvent in $\mathrm{C}_{18}$ solid phase extraction (SPE) cartridges for GSE PACs [6]. Therefore, the feces sample was cleaned up and extracted by liquidsolid extraction and the extracts were subjected to SPE. Recently, Oasis ${ }^{\circledR}$ hydrophilic-lipophilic balanced (HLB) cartridges have been widely used for polyphenolic compounds extraction [43]. The stationary phase of HLB is a reverse-phase sorbent, a polystyrene divinylbenzene copolymer and it is suitable for acids, bases and neutrals compounds. Additionally, it has been reported that 96-well plates were used for sample cleanup including plasma and urine [46]. Some of the advantages of using conventional SPE cartridges over 96-well plates were high throughput sample processing and less usage of organic solvents.

Besides SPE, liquid-liquid extraction can be used for sample extraction or as a cleanup method. This technique is suitable for lipophilic compounds extraction and solvents that commonly used were ethyl ether or ethyl acetate. High molecular weight polyphenolic compounds were extracted using aqueous organic solvent including methanol and $70 \%$ aqueous acetone. It has been reported that acidification of the extraction solvent can increase the extractability of PACs since acid helps to break the bonds between PACs and polar matrices [31].

The spike recoveries of phenolic metabolites in biological samples were varied and ranged from $80-100 \%$ in urine [46] to $50-115 \%$ in feces [6]. It has been observed that the higher the DP of PACs, the lower the spike recovery. These lower recovery rates are likely due to increasing adsorption of the larger oligomers to the complex matrix present in the feces [6].

Analytical separation and identification techniques for polyphenolic compounds includes thin layer chromatography (TLC), gas chromatography (GC) and/or liquid chromatography (LC) coupled to various mass spectrometry such as single quadrupole, triple quadruople, ion trap, time-of-flight (TOF) and nuclear magnetic resonance (NMR) $[4,15,26,29]$. The chromatographic separations were mostly performed using HPLC while GC was used for separation of low molecular weight or volatile polyphenolic compounds [14]. A reverse phase-LC method was able to separate flavan-3-ol monomer up to trimers and DP $>3$ will elute as a broad and unresolved peak at the end of the run.

Nonetheless, normal phase liquid chromatography (NP-LC) coupled with a fluorescence detector was able to separate and quantify individual PACs up to decamers [4].

A soft ionization source such as electrospray ionization (ESI) was commonly used for identifying polyphenol metabolites. Besides ESI, other ionization sources including atmospheric pressure chemical ionization (APCI) or atmospheric pressure photoionization (APPI) has been used for detecting small metabolites [29]. Both positive $[\mathrm{M}+\mathrm{H}]^{+}$or negative $[\mathrm{M}-\mathrm{H}]^{-}$ion mode can be used, although negative ion mode yields ions more effectively, due to the weak acidic nature of PACs [15].

The application of LC-MS/MS for quantitation of polyphenol compounds have been shown by others [4, 30, 46]. For metabolite identification, LC-MS/MS operating in multiple-reaction monitoring (MRM) offered high selectivity and sensitivity allowing detection of metabolites in biological samples at low concentration.

\section{Conclusions}

The adsorption and metabolism of proanthocyanidins is only partially understood, largely due to the amphilic physical properties, the complex mixtures of the natural sources and very low adsorption rates. Consequently, analytical approaches to study these substances are complex, but highly sensitive LC-MS systems capable of ionizing high molecular weight compounds are making the questions more approachable. The other complicating factor is that since the 
adsorption in the intestine is very ineffective, the compounds are largely metabolized in the colon, meaning that compounds may be important to gut chemistry, but also that the metabolites are highly fragmented by microbial action and thus more difficult to associate with the PACs. One of the most pressing questions is whether or not any PAC's remain intact in the colon, and if so, what is the concentration of these substances.

\section{Acknowledgments}

The authors would like to thank our collaborator Patricia I. Oteiza at UC Davis for her sound advice and Polyphenolics Inc for financial support.

\section{References}

[1] Appeldoorn MM, Vincken J-P, Aura A-M, Hollman $\mathrm{PCH}$, Gruppen H. Procyanidin dimers are metabolized by human microbiota with 2-(3,4-dihydroxyphenyl)acetic acid and 5-(3,4-dihydroxyphenyl)- $\gamma$-valerolactone as the major metabolites. J Agric Food Chem. 2009;57:1084-92.

[2] Appeldoorn MM, Vincken J-P, Gruppen H, Hollman PCH. Procyanidin dimers a1, a2, and b2 are absorbed without conjugation or methylation from the small intestine of rats. The Journal of Nutrition. 2009;139:1469-73.

[3] Baba S, Osakabe N, Natsume M, Muto Y, Takizawa T, Terao J. In vivo comparison of the bioavailability of (+)-catechin, (-)epicatechin and their mixture in orally administered rats. The Journal of Nutrition 2001;131:2885-91.

[4] BuendíA BA, Gil MAI, Tudela JA, Gady AL, Medina JJ, Soria C, LóPez JM, TomáS-BarberáN, FA. Hplc-ms analysis of proanthocyanidin oligomers and other phenolics in 15 strawberry cultivars $\dagger$. J Agric Food Chem. 2009;58:3916-26.

[5] Fraga CG, Oteiza PI. Dietary flavonoids: Role of (-)epicatechin and related procyanidins in cell signaling. Free Radical Biology and Medicine. 2011;51(4):813-23. ISSN 0891-5849

[6] Choy YY, Jaggers GK, Oteiza PI, Waterhouse AL. Bioavailability of intact proanthocyanidins in the rat colon after ingestion of grape seed extract. Journal of Agricultural and Food Chemistry. 2012;61:121-7.

[7] Clifford MN. Diet-derived phenols in plasma and tissues and their implications for health. Planta Med 2004;70:1103-14.

[8] Daniele Del Rio AR-M, Jeremy PE, Spencer, Massimiliano T, Gina B, Alan C. Dietary (poly)phenolics in human health: Structures, bioavailability, and evidence of protective effects against chronic diseases. Antioxid Redox Signal. 2013;18:1818-92.

[9] Deprez S, Brezillon C, Rabot S, Philippe C, Mila I, Lapierre C, Scalbert A. Polymeric proanthocyanidins are catabolized by human colonic microflora into low-molecular-weight phenolic acids. J Nutr. 2000;130:2733-8.
[10] Donovan JL, Manach C, Rios L, Morand C, Scalbert A, Remesy C. Procyanidins are not bioavailable in rats fed a single meal containing grapeseed extract or the procyanidin dimer b3. Br J Nutr. 2002;87:299-306.

[11] Fulcrand H, Remy S, Souquet J-M, Cheynier V, Moutounet M. Study of wine tannin oligomers by on-line liquid chromatography electrospray ionization mass spectrometry. Journal of Agricultural and Food Chemistry. 1999;47:1023-8.

[12] Gonthier MP, Cheynier V, Donovan JL, Manach C, Morand C, Mila I, Lapierre C, Remesy C, Scalbert A, Microbial aromatic acid metabolites formed in the gut account for a major fraction of the polyphenols excreted in urine of rats fed red wine polyphenols. J Nutr. 2003;133:461-67.

[13] Gonthier M-P, Donovan JL, Texier O, Felgines C, Remesy C, Scalbert A. Metabolism of dietary procyanidins in rats. Free Radical Biology and Medicine. 2003;35(8):837-44.

[14] Grün CH, Van Dorsten FA, Jacobs DM, Le Belleguic M, Van Velzen EJJ, Bingham MO, Janssen H-G, Van Duynhoven JPM. Gc-ms methods for metabolic profiling of microbial fermentation products of dietary polyphenols in human and in vitro intervention studies. Journal of Chromatography B. 2008;871:212-9.

[15] Hayasaka Y, Waters EJ, Cheynier V, Herderich MJ, Vidal S. Characterization of proanthocyanidins in grape seeds using electrospray mass spectrometry. Rapid Commun Mass Spectrom. 2003;17:9-16.

[16] Holt RR, Lazarus SA, Sullards MC, Zhu QY, Schramm DD, Hammerstone JF, Fraga CG, Schmitz HH, Keen CL. Procyanidin dimer b2 [epicatechin-(4ß-8)-epicatechin] in human plasma after the consumption of a flavanol-rich cocoa. The American Journal of Clinical Nutrition. 2002;76:798-804.

[17] Howell AB, Reed JD, Krueger CG, Winterbottom R, Cunningham DG, Leahy M. A-type cranberry proanthocyanidins and uropathogenic bacterial anti-adhesion activity. Phytochemistry. 2005;66:2281-91.

[18] Jimenez-Ramsey LM, Rogler JC, Housley TL, Butler LG, Elkin RG. Absorption and distribution of 14c-labeled condensed tannins and related sorghum phenolics in chickens. J Agric Food Chem. 1994;42:963.

[19] Kahle K, Huemmer W, Kempf M, Scheppach W, Erk T, Richling E. Polyphenols are intensively metabolized in the human gastrointestinal tract after apple juice consumption. J Agric Food Chem. 2007;55:10605-14.

[20] Kaur M, Singh RP, Gu M, Agarwal R, Agarwal C. Grape seed extract inhibits in vitro and in vivo growth of human colorectal carcinoma cells. Clinical Cancer Research. 2006;12:6194-202.

[21] Li S, Sui Y, Xiao J, Wu Q, Hu B, Xie B, Sun Z. Absorption and urinary excretion of a-type procyanidin oligomers from litchi chinensis pericarp in rats by selected ion monitoring liquid chromatography-mass spectrometry. Food Chemistry. 2013;138:1536-42.

[22] Manach C, Scalbert A, Morand C, Rémésy C, Jiménez L. Polyphenols: Food sources and bioavailability. The American Journal of Clinical Nutrition. 2004;79:727-7.

[23] Manach C, Williamson G, Morand C, Scalbert A, Rémésy C. Bioavailability and bioefficacy of polyphenols in humans. I. Review of 97 bioavailability studies. The American Journal of Clinical Nutrition. 2005;81:230S-42S.

[24] Mateos-Martin ML, Perez-Jimenez J, Fuguet E, Torres JL. Non-extractable proanthocyanidins from grapes are a source 
of bioavailable (epi)catechin and derived metabolites in rats. Br J Nutr. 2012;108:290-7.

[25] Mateos-Martín ML, Pérez-Jiménez J, Fuguet E, Torres JL. Profile of urinary and fecal proanthocyanidin metabolites from common cinnamon (cinnamomum zeylanicum 1.) in rats. Molecular Nutrition \& Food Research. 2012;56:671-5.

[26] Miniati E. Assessment of phenolic compounds in biological samples. Ann Ist Super Sanitá 2007:43:362-8.

[27] Monagas M, Urpi-Sarda M, Sanchez-Patan F, Llorach R, Garrido I, Gomez-Cordoves C, Andres-Lacueva C, Bartolome B. Insights into the metabolism and microbial biotransformation of dietary flavan-3-ols and the bioactivity of their metabolites. Food \& Function. 2010;1:233-53.

[28] Ottaviani JI, Kwik-Uribe C, Keen CL, Schroeter H. Intake of dietary procyanidins does not contribute to the pool of circulating flavanols in humans. Am J Clin Nutr. 2012;95:851-8.

[29] Prasain JK, Barnes S. Metabolism and bioavailability of flavonoids in chemoprevention: Current analytical strategies and future prospectus. Molecular Pharmaceutics. 2007;4:84664.

[30] Prasain JK, Peng N, Dai Y, Moore R, Arabshahi A, Wilson L, Barnes S, Michael Wyss J, Kim H, Watts RL. Liquid chromatography tandem mass spectrometry identification of proanthocyanidins in rat plasma after oral administration of grape seed extract. Phytomedicine. 2009;16:233-43.

[31] Prior RL, Gu L. Occurrence and biological significance of proanthocyanidins in the american diet. Phytochemistry. 2005;66:2264-2280.

[32] Rios LY, Bennett RN, Lazarus SA, Rémésy C, Scalbert A, Williamson G. Cocoa procyanidins are stable during gastric transit in humans. The American Journal of Clinical Nutrition. 2002;76:1106-1110.

[33] Rossi M, Negri E, Parpinel M, Lagiou P, Bosetti C, Talamini R, Montella M, Giacosa A, Franceschi S, La Vecchia C. Proanthocyanidins and the risk of colorectal cancer in italy. Cancer Causes and Control. 2010;21:243-50.

[34] SáNchez-PatáN F, Monagas MA, Moreno-Arribas MV, Bartolomé BA. Determination of microbial phenolic acids in human faeces by uplc-esi-tq ms. Journal of Agricultural and Food Chemistry. 2011;59:2241-47.

[35] Sano A, Yamakoshi J, Tokutake S, Tobe K, Kubota Y, Kikuchi M. Procyanidin b1 is detected in human serum after intake of proanthocyanidin-rich grape seed extract. Biosci, Biotechnol, Biochem. 2003;67:1140.

[36] Serra A, Maciá A, Romero M-P, Salvadó M-J, Bustos M, Fernández-Larrea J, Motilva M-J. Determination of procyanidins and their metabolites in plasma samples by improved liquid chromatography-tandem mass spectrometry. Journal of Chromatography B. 2009;877:1169-76.

[37] Shoji T, Masumoto S, Moriichi N, Akiyama H, Kanda T, Ohtake Y, Goda Y. Apple procyanidin oligomers absorption in rats after oral administration: Analysis of procyanidins in plasma using the porter method and high-performance liquid chromatography/tandem mass spectrometry. J Agric Food Chem. 2006;54:884-92.

[38] Singletary KW, Meline B. Effect of grape seed proanthocyanidins on colon aberrant crypts and breast tumors in a rat dual-organ tumor model. Nutr Cancer. 2001;39:252-8.

[39] Stalmach A, Mullen W, Steiling H, Williamson G, Lean MEJ, Crozier A. Absorption, metabolism, and excretion of green tea flavan-3-ols in humans with an ileostomy. Molecular Nutrition \& Food Research. 2010;54:323-34.

[40] Terrill TH, Waghorn GC, Woodley DJ, Mcnabb WC, Barry $\mathrm{TN}$. Assay and digestion of 14c-labeled condensed tannins in the gastrointestinal tract of sheep. Br J Nutr. 1994;72:467-77.

[41] Terry P, Giovannucci E, Michels KB, Bergkvist L, Hansen H, Holmberg L, Wolk A. Fruit, vegetables, dietary fiber, and risk of colorectal cancer. J Natl Cancer Inst. 2001;93:525-33.

[42] Theodoratou E, Kyle J, Cetnarskyj R, Farrington SM, Tenesa A, Barnetson R, Porteous M, Dunlop M, Campbell H. Dietary flavonoids and the risk of colorectal cancer. Cancer Epidemiology Biomarkers \& Prevention. 2007;16:684-93.

[43] Touriño S, PéRez-JiméNez J, Mateos-MartíN MAL, Fuguet E, Vinardell MAP, Cascante M, Torres JLS. Metabolites in contact with the rat digestive tract after ingestion of a phenolic-rich dietary fiber matrix. Journal of Agricultural and Food Chemistry 2011;59:5955-63.

[44] Tsang C, Auger C, Mullen W, Bornet A, Rouanet J-M, Crozier A, Teissedre P-L. The absorption, metabolism and excretion of flavan-3-ols and procyanidins following the ingestion of a grape seed extract by rats. Br J Nutr. 2005;94:170-181.

[45] Urpi-Sarda M, Monagas M, Khan N, Lamuela-Raventos R, Santos-Buelga C, Sacanella E, Castell M, Permanyer J, AndresLacueva C. Epicatechin, procyanidins, and phenolic microbial metabolites after cocoa intake in humans and rats. Analytical and Bioanalytical Chemistry. 2009;394:1545-56.

[46] Urpi-Sarda M, Monagas M, Khan N, Llorach R, LamuelaRaventós RM, Jáuregui $\mathrm{O}$, Estruch R, Izquierdo-Pulido M, Andrés-Lacueva C. Targeted metabolic profiling of phenolics in urine and plasma after regular consumption of cocoa by liquid chromatography-tandem mass spectrometry. Journal of Chromatography A. 2009;1216:7258-67.

[47] Velmurugan B, Singh RP, Agarwal R, Agarwal C. Dietaryfeeding of grape seed extract prevents azoxymethane-induced colonic aberrant crypt foci formation in fischer 344 rats. Molecular Carcinogenesis. 2010;49:641-52.

[48] Vivas N, Glories Y, Pianet I, Barbe B, Laguerre M. A complete structural and conformational investigation of procyanidin a2 dimer. Tetrahedron Letters. 1996;37:2015-18.

[49] Wang Y, Chung S-J, Song WO, Chun OK. Estimation of daily proanthocyanidin intake and major food sources in the u.S. Diet. The Journal of Nutrition. 2011;141:447-52.

[50] Yang CS, Chen L, Lee MJ, Balentine D, Kuo MC, Schantz SP. Blood and urine levels of tea catechins after ingestion of different amounts of green tea by human volunteers. Cancer Epidemiology Biomarkers \& Prevention. 1998;7:351-4. 